\title{
Interprète simultané par rapport à l'image
}

\section{Simultaneous interpreter in relation to the image}

\author{
Justyna Woroch \\ Uniwersytet im. Adama Mickiewicza w Poznaniu \\ j.woroch@amu.edu.pl
}

\begin{abstract}
The aim of this article is to signal the possible relationships between the simultaneous interpreter and the image in its widest sense. In this communicative situation, attention is first paid to the text of the oral presentation, the meaning of which is conveyed by the interpreter. However, this meaning is also made up of visual elements that the interpreter faces and which make the interpretation inter-semiotic and multimodal. Three different types of simultaneous interpreter/image relationships will be outlined: firstly, the whole interpreting communicative situation can become an image if remote interpretation is necessary, secondly, it is the speaker who can be understood as an image, thirdly, the speaker can refer to previously prepared images. Then, by means of a pilot study, it will be checked whether and how experienced simultaneous interpreters relate the speaker to the picture when the picture presented is not redundant with what is being said, but complements the speech. The main question is whether and how it will be verbalized. Possible ways of extending the study will also be discussed.
\end{abstract}

Keywords: simultaneous interpreting, visual element, image, verbalization

\section{INTRODUCTION}

Si nous réfléchissons à la situation de communication spécifique qui est celle de l'interprétation de conférence, et surtout de l'interprétation simultanée, notre attention se porte tout d'abord sur le texte de l'exposé oral, dont le sens est transmis par l'interprète. Cependant, ce sens est également composé d'éléments visuels auxquels l'interprète a recours et qui rendent l'interprétation intersémiotique et multimodale.

Notre article a pour but de signaler les différentes relations existant entre l'interprète simultané et l'image au sens large du terme, pour ensuite se focaliser sur les 
éléments visuels accompagnants les propos de l'orateur durant une conférence et leur possible interprétation. Par le biais d'une analyse descriptive découlant d'une étude pilote de cas, nous comptons percevoir les moyens de véhiculer (ou non) la composante visuelle du message donné par l'orateur. Cette étude pilote s'inscrit dans le cadre d'un projet à visée plus large, mené individuellement et sans financement institutionnel quelconque, ayant pour but d'examiner les pistes didactiques potentielles visant à sensibiliser les adeptes de l'interprétation de conférence à la complexité de la tâche de l'interprétation. L'accent est mis sur l'apport des éléments visuels faisant partie de cette communication, et s'inspire des acquis des traducteurs audiovisuels face aux éléments communs à ces situations variées où on a affaire à la transmission du sens véhiculé par différents canaux (cf. Tomaszkiewicz, 2006). Ceci est d'autant plus important que de nos jours les présentations visuelles sont fréquentes lors des réunions multilingues faisant appel aux interprètes (cf. Berber Irabien, 2010 ; Baxter, 2016).

Tout d'abord, il convient de mentionner un aspect de l'interrelation entre l'interprète simultané et l'image, celui de l'interprétation à distance qui met en œuvre le processus intéressant de "l'image dans l'image ", où l'interprète est confronté à une image vidéo de la situation de communication dans laquelle il prend place. Dans ce cadre relativement inconfortable pour les interprètes avant la crise épidémiologique de 2019, l'interprète se trouve littéralement confronté à l'image, celle des visioconférences. Même si les recherches sur le sujet sont en cours depuis plusieurs années (Mouzourakis, 1996, 2006 ; Moser-Mercer, 2005a, 2005b ; Braun, 2006, 2012, 2016 ; Braun \& Taylor, 2012 ; Roziner \& Shlesinger, 2010 ; Peterc 2020), la situation de crise sanitaire a accéléré la mise en pratique de certaines solutions. L'interprète perçoit donc une image très complexe qui pourrait faire l'objet d'une analyse séparée.

Ensuite, c'est l'orateur lui-même qui constitue une image en communiquant verbalement et en même temps non verbalement, avec ses auditeurs. D'où par exemple l'importance de garantir à l'interprète une bonne vue de la salle et de l'orateur (cf. Jesse et al., 2000 ; Moser-Mercer, 2005a). Si ce dernier fait un geste significatif, montre quelque chose en utilisant son corps, sa mimique, comment fera l'interprète ? Verbalisera-t-il ce mouvement, fera-t-il un commentaire sur ce geste ? Rennert (2008) observe que dans ce cas, la composante visuelle ne se limite pas à la communication non verbale de l'orateur, mais peut être étendue à une vue complète de la salle, du public et des présentations visuelles. Cependant, elle souligne que c'est la communication non verbale, en tant que partie intégrale de la communication, qui constitue une des plus riches sources d'informations supplémentaires pour l'interprète. En effet, les signaux non verbaux peuvent remplir différentes fonctions par rapport au message verbal qu'ils précèdent, accompagnent ou suivent. Ils peuvent ajouter une information, renchérir, réitérer, ou affaiblir, voire contredire ce qui est exprimé verbalement. Ils peuvent même remplacer certains mots (Poyatos, 1997).

Finalement, l'orateur peut aussi se référer à de l'image, préparée au préalable, généralement sous forme de présentation incluant éventuellement photographies, dessins, cartes, diagrammes ou des vidéos. Quelles peuvent être les différentes relations 
entre le texte du discours de l'orateur et les images qu'il visionne ? Sachant que nous assistons forcément à un décalage dans le temps et dans l'espace entre le visionnement de l'image destinée aux auditeurs et le message transmis par l'interprète, ce denier adoptera-t-il une technique spécifique pour dépasser ce problème?

Dans le cadre du présent article, nous nous limiterons à esquisser ce dernier type de relations entre l'interprète simultané et le texte de l'orateur accompagné d'images, en illustrant notre propos par une expérience menée à petite échelle, dans le cadre d'une étude pilote, avec la participation de quatre interprètes de conférence professionnels, pour percevoir leurs moyens de véhiculer la composante visuelle du message transmis par l'orateur. Nous caractériserons davantage les types d'éléments visuels choisis dans la partie consacrée à ces éléments dans l'interprétation simultanée.

\section{IMAGE VISIONNÉE PAR L'ORATEUR}

Il est donc important pour l'interprète de percevoir non seulement l'orateur, tout comme les autres éléments prenant part au processus de communication, mais aussi, de voir et de posséder les informations visuelles qu'il restitue d'une autre façon aux auditeurs.

Les chercheurs ont démontré que l'information verbale visuelle (Pöchhacker, 1994), c'est-à-dire les statistiques ou les citations inclues habituellement dans les présentations PowerPoint, sont très souvent présentées à rapidement. Quand l'information est redondante, l'interprète peut opter pour une technique selon laquelle il ne répète pas ce que le public peut voir de lui-même, mais recourt à une ellipse du type «Comme vous pouvez le voir sur cette diapo » (Bühler, 1980 ; Alonso Bacigalupe, 1999 ; Eder 2003, in Rennert 2008). Cependant, comme le note Baxter (2016), « il s'agit de phénomènes marginaux $[\ldots]$ n'étant qu'une solution occasionnelle de dernier recours plutôt qu'une partie intégrante de la technique de base de l'interprétation, l'usage répété pouvant entraîner une certaine lassitude de la part du public qui, pour une raison quelconque (par exemple, déficience visuelle, diapositives illisibles, diapositives dans une langue qu'il ne connaît pas, etc.) peut ne pas être en mesure de «voir ce qu'il y a dans l'image» et qui s'attend à recevoir de la part de l'interprète, via son casque, les informations dont il a besoin, concernant le contenu du discours original» (p. 10, trad. J.W.). À cela s'ajoute la question de la synchronisation de ce que le public voit avec ce que l'interprète lui transmet. Une recherche pilote de Baxter (2016) a fait émerger un phénomène intéressant à exploiter : contrairement à l'hypothèse initiale de l'auteur, le fait que l'orateur se serve d'une présentation PowerPoint augmenterait le décalage de l'interprète par rapport à l'orateur et ses diapositives. Ceci peut être en partie expliqué par le fait que l'attention de l'interprète est partagée entre le texte source, délivré à l'oral, et les diapositives, à la recherche d'informations relatives à ce qui est dit. Une autre recherche, plus récente, de Sainsbury (2020) soulève une autre question très intéressante, celle de l'impact de la densité lexicale des diapositives sur l'interprétation simultanée. Tout ce qui précède fait référence à deux approches principales concernant 
l'interprétation simultanée avec texte : le modèle des efforts de Gile (Gile, 2009 ; Gile, 2018) et le modèle de la charge cognitive de Seeber (Seeber, Kerzel, 2011 ; Seeber, 2017 ; Seeber et al., 2019). Ces modèles se concentrent sur la coprésence du texte durant l'interprétation simultanée qui peut être soit visionné sous forme de présentations, soit mis à disposition des interprètes sous forme de discours écrits au préalable.

Ce qui nous intéresse, par contre, se résume à un type spécifique de relations entre le discours prononcé par l'orateur et ce qu'il fait visionner durant sa conférence. Nous pensons aux cas particulier des présentations PowerPoint auxquelles se réfère l'orateur, incluant non seulement un texte ou des schémas, mais surtout des photos ou des images véhiculant un sens voulu par l'orateur. Dans les cas où l'image n'est pas redondante par rapport à ce que l'orateur transmet, que fera l'interprète simultané ? Sera-t-il en mesure d'en verbaliser le contenu ?

\section{ÉLÉMENTS VISUELS DANS L'INTERPRÉTATION SIMULTANÉE : UNE ÉTUDE PILOTE DE CAS}

Notre étude pilote vise à examiner comment l'interprète simultané professionnel rendra la relation entre le texte de l'orateur et l'image présentée, lorsque celle-ci ne reprend pas son contenu mais le complète d'une certaine façon. À cette fin, nous avons soigneusement choisi un discours authentique de dix-huit minutes, prononcé en polonais lors d'une conférence TEDx. Ce discours est disponible en libre accès sur la page web de l'évènement (https://www.youtube.com/watch? $\mathrm{v}=\mathrm{sHY} 7 \mathrm{TdnWFuQ}$ ). Il donne des conseils pour réaliser de bonnes présentations et se caractérise par le fait qu'il contient plusieurs types de références à ce type d'images, qui seront décrites par la suite. Nous avons opté pour un sujet pas trop spécialisé se prêtant en même temps aux illustrations à l'aide d'images. Nous avons demandé à 4 professionnels de l'interpréter simultanément, dans les conditions d'expérimentation, c'est-à-dire à domicile, installés devant l'ordinateur et enregistrant leurs propos de façon autonome. Nous avons ainsi reçu quatre enregistrements de l'interprétation simultanée de ce discours, deux en français et deux en anglais. Le choix de l'interprétation vers la langue B des interprètes était motivé d'un côté, par l'hypothèse qu'une meilleure compréhension apparaît lors de l'interprétation d'un discours prononcé en langue maternelle, et de l'autre côté, par l'accessibilité de professionnels favorables à cette expérimentation, travaillant tous dans les deux directions linguistiques respectives.

Le déroulement de cette activité a été planifié comme suit:

1. Demande par écrit de l'acceptation de la participation à l'étude pilote, garantie de l'anonymat des résultats obtenus et de la confidentialité des données des participants.

2. Après l'accord obtenu, envoi du protocole de l'étude pilote : consigne de ne pas écouter le discours avant la réalisation effective de l'interprétation, afin de garantir que soit renvoyée pour analyse la première interprétation ; lien vers un 
autre discours de ce même orateur, discutant un sujet similaire, en vue de faire connaître le contexte et la façon dont parle l'orateur (le débit, le rythme, le lexique spécifique) ; petit glossaire de mots spécialisés et de noms propres apparaissant dans le discours (sans propositions d'équivalents dans les deux langues mentionnées) accompagné d'informations contextuelles sur le discours (date, lieu, thème de la conférence, titre du discours, etc.) ; rappel que l'activité a pour but d'observer les relations avec l'image et non pas l'évaluation de la fréquence des erreurs par exemple (pas plus d'informations pour ne pas impacter les résultats).

3. Consigne finale : Interprétez le discours comme vous le feriez si vous étiez un interprète simultané TEDx pour un public francophone/anglophone.

4. Aussi bien le discours original que les interprétations fournies ont été transcrites par les soins de l'auteur.

Nous nous sommes interrogée sur l'éventuel reproche du caractère artificiel de la situation arrangée, sachant que l'image vidéo ne donnait pas forcément accès aux informations visuelles qui seraient disponible à l'interprète en situation directe. Anderson (1994) souligne ce point, en évoquant les recherches empiriques sur l'interprétation de conférence. Néanmoins, vu la situation actuelle dans le monde de l'interprétation de conférence et des recherches précitées sur l'interprétation à distance, nous trouvons cela tout à fait légitime.

Pour les besoins de l'analyse qui suit, nous avons sélectionné des exemples du discours, dans lesquels la relation entre le texte prononcé et les images présentées peut être décrite, selon Tomaszkiewicz, comme une relation de complémentarité (2006, p. 60). Cela signifie que certains éléments du contenu sont exprimés verbalement, tandis que d'autres le sont visuellement. Comme le précise Tomaszkiewicz (2006, p. 60), il n'est pas possible de comprendre l'ensemble du message sans comprendre les signes de chacune de ces couches et sans déchiffrer comment, ensemble, ils véhiculent du sens. En d'autres termes, les images font partie du discours analysé. Sans elles, le message du texte serait déformé. Un élément supplémentaire, ajoutant aux difficultés potentielles de l'interprétation ultérieure de ce type de texte, dans lequel le rôle des images qui l'accompagnent est mise en évidence, est la recherche d'un effet humoristique par l'orateur, car cet effet est obtenu précisément par la juxtaposition délibérée d'éléments visuels et verbaux. Selon la définition généralement admise du comique, son essence consiste à révéler une contradiction ou un contraste surprenant, résultant de la présentation de personnes ou de situations différant des attentes du destinataire, ce qu'on explique plus loin dans l'analyse.

\section{ANALYSE ET DISCUSSION}

Dans le premier exemple choisi, l'effet humoristique se produit lorsque l'orateur explique qu'une diapositive a trois rôles à remplir, entre autres, celui d'assurer la 
chute. Juste après cette explication, il l'illustre avec une petite histoire accompagnée d'une image. Le référent présent sur l'image est culturellement ancré et son décodage repose sur un savoir commun pour les utilisateurs de la langue polonaise :

Traduction en français de l'original polonais : Et cette diapositive est censée vous surprendre. Si jamais un jour vous vous sentiez inutile dans la vie, pensez à cet endroit (fig. 2).

\section{Image accompagnante :}

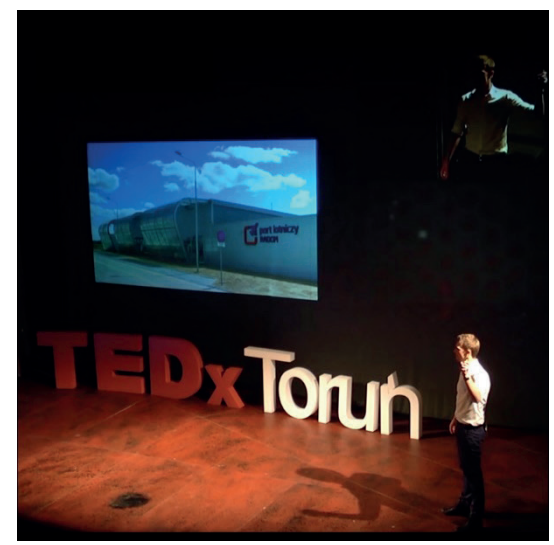

Fig. 2. Capture d'écran du discours analysé

Explication du contexte : Déjà au stade de sa construction, les experts ont suggéré que l'aéroport de Radom présenté sur l'image, serait probablement un échec financier. À cette époque, de nombreux mèmes sont apparus sur Internet, faisant référence à cet investissement sans doute peu judicieux, forcé par les autorités locales. L'orateur ne nomme pas l'endroit visionné sur l'image, il le désigne à l'aide d'un déictique spatial et d'un hyperonyme (to miejsce), cependant cette image est accompagnée d'une inscription. Le public polonais qui ne connaîtrait pas l'endroit visé par l'orateur et donc informé par cette inscription. Notons de passage que la relation entre le texte visible sur la photo et ce qu'elle représente est celle de l'équivalence, selon la typologie proposée par Tomaszkiewicz (2006). L'orateur procède donc à une comparaison et à une métaphore implicite : nous pouvons nous sentir parfois inutiles, mais ce n'est rien par rapport l'inutilité de cet aéroport de Radom.

Analysons si le même effet de sens peut se reproduire dans l'interprétation. Autrement dit, l'interprète a-t-il la possibilité de verbaliser tout ce qui se cache derrière cette image ?

Int. 1 PL-FR : La diapo doit vous surprendre. Si une fois dans la vie vous vous sentez inutile, vous pourrez penser à cet endroit.

Int. 2 PL-FR : La diapo doit vous surprendre. Si quelquefois vous vous sentez inutile, regardez là. 
Int. 1 PL-EN : And it should surprise you. If you feel useless, think about this place. And what's shown is the airport in Radom.

Int. 2 PL-EN : The slide is supposed to change the way your audience sees you. So if ever in your life you feel useless think about this.

Parmi les quatre propositions d'interprétation, une seule (Int. 1 PL-EN) tente de développer davantage ce qui est dit dans l'original, dans sa couche verbale et est exprimée grâce à un déictique spatial : Pomyślcie o tym miejscu / Pensez à cet endroit. Les interprétations 1 et 2 PL-FR et Int. 2 PL-EN reproduisent uniquement les déictiques, les pronoms démonstratifs servant à renvoyer à l'image. Cependant le sens du message peut être illisible. Même dans le cas de la première proposition évoquée, où l'interprète verbalise qu'il est question de l'aéroport de Radom, un récepteur étranger pourrait ne pas capter le sens et l'effet humoristique d'une telle combinaison car l'histoire évoquée grâce à cette image n'est pas universellement connue, ni annoncée auparavant dans le discours de l'orateur.

Dans le deuxième exemple choisi, la relation du sens entre le discours de l'orateur et l'image qu'il présente est aussi du type humoristique, mais construite d'une façon différente car cette fois-ci le référent est universellement connu. L'orateur explique qu'une bonne diapositive fonctionne comme un panneau publicitaire $4 \times 3$ :

Traduction en français de l'original polonais : Les maîtres de scène savent que la diapositive est un dérivé du panneau d'affichage. Et c'est à cela que ressemble un bon panneau d'affichage (fig. 3). Cependant, ils se rendent compte de ce qui se passerait si les créateurs de panneaux d'affichage avaient soudain (la mauvaise) idée de prendre des leçons chez les créateurs de diapos (fig. 4). À en juger par votre réaction, je pense avoir touché dans le mille.

\section{Images accompagnantes :}

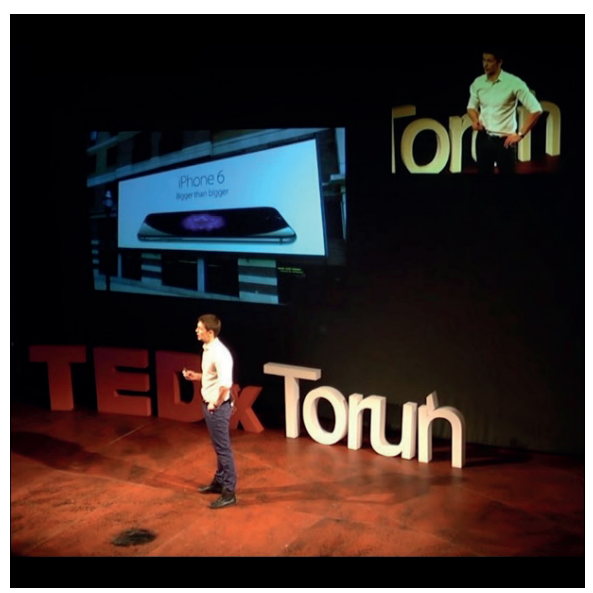

Fig. 3. Capture d'écran du discours analysé

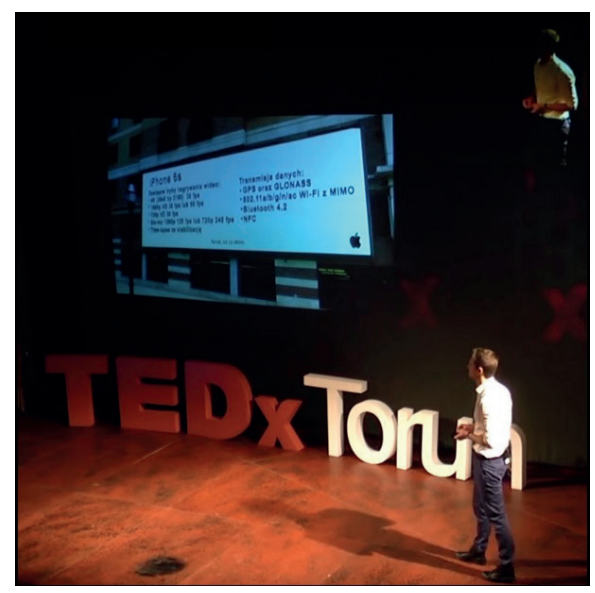

Fig. 4. Capture d'écran du discours analysé 
Explication du contexte : L'humour se produit lorsque les deux diapositives (fig. 3 et fig. 4) se suivent. L'orateur n'explique pas verbalement le contraste visible des deux images renvoyant à un même objet, qui est un Iphone6. Cependant, la seconde diapositive fait référence implicite au concept de «bloc de texte», constituant une des caractéristiques déconseillées dans les présentations.

Analysons si ce sens est reproduit dans l'interprétation et si oui, par le biais de quels moyens.

Int. 1 PL-FR : Les intervenants de TED savent que la présentation, le diapo, est comme un panneau d'affichage. Et voilà un bon panneau d'affichage. Vous pouvez imaginer ce qui arriverait si les créateurs des panneaux d'affichages prenaient l'exemple sur les créateurs des diapos. À en observer vos réactions je pense d'avoir atteint l'objectif, le but.

Int. 2 PL-FR : Les maîtres de la scène savent qu'une diapo est comme une billboard, un grand panneau de publicité. Et si par exemple les créateurs des billboards voulaient imiter ceux qui font les diapos pour la présentation? Bon, en regardant votre réaction, je pense que j'ai réussi à vous faire la différence.

Int. 1 PL-EN : Well, stage masters know that a slide is a derivative of a billboard. This is what a good billboard looks like. They also realize what would happen if billboard designers came to a conclusion that they will take some lessons with slide designers. Well I think I make my point judging by your response.

Int. 2 PL-EN : Masters know that a slide is like a billboard. This is what a good billboard looks like. But they think what would happen if billboard designers figure out that they would learn from slideshow designers. Hearing your reaction I think I arrived at my point.

Parmi les propositions d'interprétations il y en a trois (Int. 1 PL-FR, Int. 1 PLEN, Int. 2 PL-EN) qui reprennent presque littéralement l'annonce de la première des deux diapositives à avoir été présentées par l'orateur. Grâce à l'emploi des déictiques (comme dans l'original), le récepteur étranger potentiel est plus clairement informé qu'il doit focaliser son attention sur l'image présentée (celle représentant la photo du téléphone portable, donc un «panneau d'affichage réussi »). Dans le cas de l'Int. 2 PL-FR, ceci paraît moins clair car la référence à l'image n'est pas verbalisée. Reste à savoir si la synchronisation de l'image montrée par l'orateur avec les propos prononcés par l'interprète advient au bon moment. Ceci est une de nos conclusions de travail, tirée de cette étude pilote à modeste échelle, qu'une mesure de synchronisation plus poussée, par le biais d'un logiciel spécialisé, mériterait d'être effectuée pour savoir si ce à quoi l'interprète se réfère (la diapositive en question) est toujours visible pour les auditeurs, sachant qu'un décalage se produit toujours entre la parole de l'orateur et celle de l'interprète (il faut mentionner ici les résultats d'une recherche empirique de Baxter (2016) à ce propos, dans l'idée de les prolonger, en ajoutant cet aspect purement visuel qui nous intéresse).

Le troisième cas que nous nous proposons d'observer consiste en une interrelation provoquant également un effet humoristique, mais cette fois-ci, basée aussi bien 
sur un élément visuel renvoyant à un référent culturellement ancré, qu'à un second, universellement connu et auquel l'orateur renvoie en même temps. Nous nous demandons si et laquelle de ces composantes sera verbalisée par l'interprète :

Traduction en français de l'original polonais : La police de caractère qui apparaît ici (fig. 5), ou Lobster, est une très belle police [...] mais je ne voudrais certainement pas voir la composition du Conseil des ministres rédigée avec cette police, à fortiori celle de mon propre gouvernement, et pourtant je l'ai vue (fig. 6). C'est authentique. Je n'ai rien inventé. Quand je regarde cette diapositive, j'ai envie de dire " pour moi, il y aura un Kopacz Burger, un Szczurek cuit au four et un Schetyna frit à point». Et bien sûr, Internet n'oublie pas ces choses-là (fig. 7) et donc voilà ce que ça donne.

\section{Images accompagnantes :}

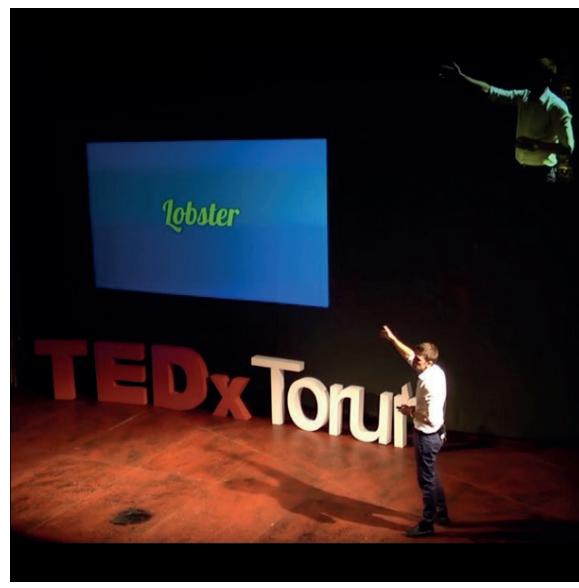

Fig. 5. Capture d'écran du discours analysé

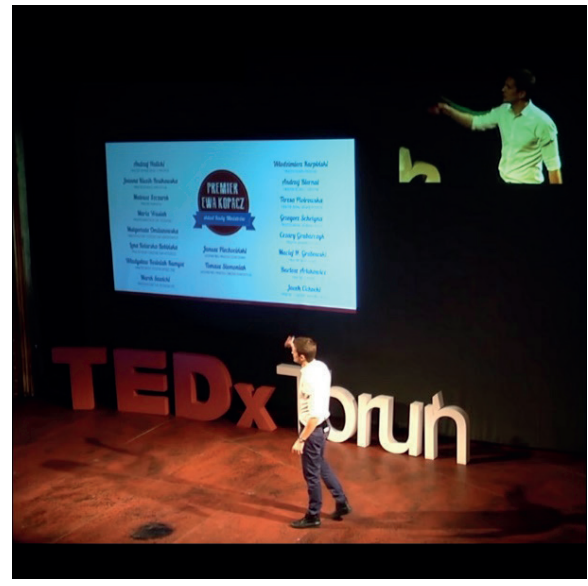

Fig. 6. Capture d'écran du discours analysé

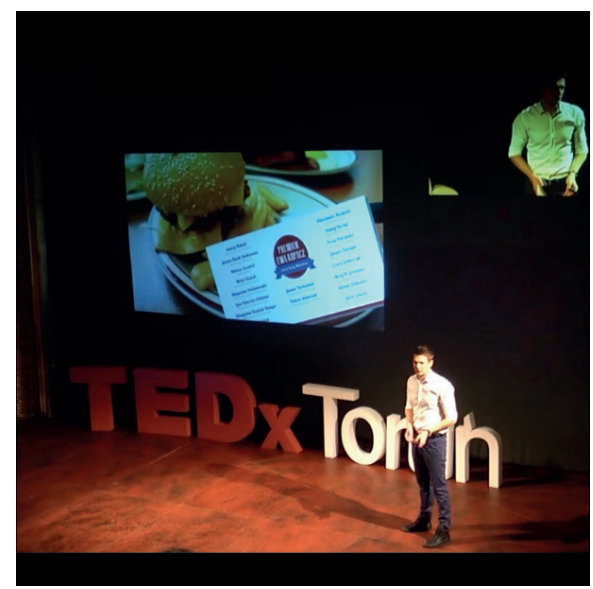

Fig. 7. Capture d'écran du discours analysé 
Explication du contexte : L'orateur explique verbalement et en même temps présente ce à quoi ressemble la police Lobster (fig. 5). L'effet humoristique se produit lorsqu'il continue en évoquant la situation hypothétique dans laquelle il serait obligé de regarder la composition du gouvernement écrite avec cette police. Ensuite il présente une diapositive avec une image trouvée sur internet (fig. 6) pour commenter la réaction des internautes sur le choix maladroit de cette police (fig. 7).

Int. 1 PL-FR : Lobster. C'est très beau. [...] Mais je ne voudrais surtout pas regarder une liste de mon gouvernement, les membres de mon gouvernement, écrits avec ce caractère, et quand même je l'ai vu, c'est une version authentique. En le regardant, j'ai envie de commander un Kopacz burger, pour expliquer, Kopacz c'est une ancienne ministre polonaise de la santé. Bien sûr Internet n'oublie pas ce genre de choses.

Int. 2 PL-FR : Lobster. C'est une police à caractère très jolie. [...] Mais je veux pas voir le conseil des ministres donné dans ce police. Mais j'étais obligé quand même de le voir. C'est authentique, je l'ai pas inventé. Quand je regarde, bon, moi je veux un Kopacz burger, Szczurek en salade et mi saignant Schetyna. Et bien sûr les Internet, Internet ne nous oublie pas.

Int. 1 PL-EN : It's Lobster. Nice font, isn't it? [...] But I wouldn't like to see the composition of the Council of Ministers of my own government printed in this particular way, using this font. And I had to see it unfortunately. This is something genuine. When I'm looking at it, so this is going to be like Kopacz Burger, and then Szczurek casserole, and medium rare Schetyna. Well, the Internet does not forget this, so there we go. This is what it looks like.

Int. 2 PL-EN : This one, Lobster. It's a very nice font. [...] But I wouldn't like to see a list of new ministers in this font, especially if it was about my government. But I had to, unfortunately. This is a real thing, I didn 't make this up. So the prime minister is a burger and then I had one minister roasted and another minister fries. Of course, the Internet never forget. So this is what it looks like.

Tous les interprètes ont mentionné le nom propre figurant sur la première diapositive de cette série, Lobster. Seulement une interprétation (Int. 2 PL-EN) recourt à un déictique («this one »), étant le plus près de l'original. Ce qui est intéressant à observer dans ce dernier exemple, c'est la façon dont les interprètes rendent le passage qui se réfère à un élément déjà connu (la police de caractère dont on parle) et la réalité purement polonaise (les noms propres des membres du gouvernement). Trois interprétations le font en utilisant les noms propres polonais et une (Int. 1 PL-FR) en expliquant la fonction de la personne publique évoquée ; une autre (Int. 2 PL-EN) procède à une généralisation, en omettant les noms propres en question qui sont visibles sur l'écran. 


\section{CONCLUSION}

Il ne fait aucun doute que l'image évoquée dans le titre de cet article peut être comprise de plusieurs façons. Après avoir considéré les relations possibles entre l'interprète simultané et l'image dans son sens le plus large, trois types de regards possibles sur cette situation communicative ont été identifiés : l'image, conçue au sens large comme un message visuel dans l'interprétation à distance, l'orateur qui constitue une certaine image pour l'interprète, et enfin l'image projetée par l'orateur et sa relation avec le discours prononcé

Dans cet article, nous nous sommes intéressée à l'observation possible des techniques par lesquelles les interprètes professionnels parviennent à transmettre des relations sélectionnées entre le texte de l'orateur et l'image affichée par celui-ci, en supposant que cette relation ne soit pas redondante. Comme cette étude pilote avait pour but de vérifier quel type d'observation serait possible, sachant que ce qui est le plus facile à observer est le produit final (Rennert, 2008), nous nous sommes concentrée ensuite sur les moyens avec lesquels les interprètes simultanés professionnels traitent les relations existant entre la couche verbale et visuelle des propos de l'orateur.

Nous avons réussi à observer si les références aux images apparaissant dans le discours à interpréter étaient verbalisées. Si nous pouvons confirmer que les déictiques renvoyant aux images visionnées étaient rendus littéralement, reste à vérifier s'ils apparaissent au bon moment, c'est-à-dire s'ils sont toujours en phase avec ce que le destinataire étranger voit au moment où il entend l'interprétation. Nous tenons donc à approfondir cet examen par des prises de mesures, durant une expérimentation faite en un lieu et à un moment précis avec un groupe d'interprètes, en présence du public, des destinataires de l'interprétation, où une situation plus naturelle se produirait. Nous tenterons aussi d'élaborer un questionnaire, tant pour les interprètes que pour le public, afin d'approfondir nos réflexions sur le processus d'interprétation, et pas seulement sur le produit final. Dans le cas des interprètes, nous avons l'intention de vérifier si et quand les images ont été utiles et quand elles ont accentué la difficulté ; pour les destinataires, il serait intéressant de vérifier si le décalage n'a pas compliqué la compréhension du discours, s'ils ont saisi les moments où l'image n'était pas en phase avec la parole/le texte.

Quant aux allusions faites dans le discours aux référents culturellement ancrés, nous avons observé un manque de technique efficace pour véhiculer le sens voulu par l'orateur et pour obtenir l'effet humoristique de l'original. Ce constat est tout de même prometteur dans la perspective de continuer cette recherche, afin d'adapter certaines techniques et de sensibiliser les adeptes de l'interprétation aux relations qui existent entre le texte et l'image, inhérentes aux discours à interpréter.

Il serait finalement très intéressant de vérifier, sur un échantillon beaucoup plus large, s'il y a des différences de verbalisation de certains éléments choisis d'une langue à l'autre (cette piste étant inspirée de l'article sur la traduction des sous-titres filmiques de Baumgarten, 2008). 


\section{BIBLIOGRAPHIE}

Anderson, L. (1994). Simultaneous interpretation. Contextual and translation aspects. In S. Lambert, B. Moser-Mercer (eds.), Bridging the Gap: Empirical research in simultaneous interpretation (pp. 101-120). Amsterdam : Benjamins, https://doi.org/10.1075/btl.3.11and.

Baumgarten, N. (2008). Yeah, that's it!: Verbal Reference to Visual Information in Film Texts and Film Translations. Meta, 53 (1), 6-25, https://doi.org/10.7202/017971ar.

Baxter, R.N. (2016). Exploring the Possible Effects of Visual Presentations on Synchronicity and Lag in Simultaneous Interpreting. Sendebar. Revista de Traducción e Interpretación. Universidad de Granada, 27, 9-23. Retrieved from : https://revistaseug.ugr.es/index.php/sendebar/issue/archive.

Berber Irabien, D. (2010). Information and Communication Technologies in Conference Interpreting. Doctoral Thesis. Tarragona : Université Rovira i Virgili. Retrieved from : https://www.researchgate. net/publication/279462774_Information_and_communication_technologies_in_conference_interpreting.

Braun, S. (2006). Multimedia communication technologies and their impact on interpreting. In H. Gerzymisch-Arbogast (ed.), Proceedings of the Marie Curie Euroconferences MuTra: Audiovisual Translation Scenarios Copenhagen, 1-5 May 2006. Retrieved from : http://www.euroconferences.info/ proceedings/2006_Proceedings/2006_proceedings.html.

Braun, S. (2015). Remote interpreting. In H. Mikkelson \& R. Jourdenais (eds.), The Routledge Handbook of Interpreting (pp. 352-367). New York : Routledge.

Braun, S., Davitti, E., Dicerto, S. (2016). Handbook of Bilingual Videoconferencing. The use of Videoconferencing in Proceedings Conducted with the Assistance of an Interpreter. Retrieved from : http://www.videoconference-interpreting.net/wp-content/uploads/2016/08/AVIDICUS3_Handbook_ Bilingual_Videoconferencing.pdf.

Braun, S., Taylor, J.L. (2012). Videoconference and Remote Interpreting in Criminal Proceedings. Antwerp/Cambridge: Intersentia. Retrieved from : http://wp.videoconference-interpreting.net/?page_id $=27$.

Gile, D. (2009). Basic Concepts and Models for Interpreter and Translators Training. Revised edition. Amsterdam/Philadelphia : John Benjamins Publishing Company.

Gile, D. (2018). Research into Translation as a specialty: An analysis and recommendations. Jostrans, 30, 23-39. Retrieved from : www.jostrans.org/issue30/art_gile.pdf.

Jesse, A., Vrignaud, N., Cohen, M.M. et Massaro, D.W. (2000). The processing of information from multiple sources in simultaneous interpreting. Interpreting, 5 (2), 95-115.

Moser-Mercer, B. (2005a). Remote Interpreting: Issues of Multi-Sensory Integration in a Multilingual Task. Meta, 50 (2), 727-738, https://doi.org/10.7202/01101ar.

Moser-Mercer, B. (2005b). Remote Interpreting: The Crucial Role of Presence. Bulletin suisse de linguistique appliquée, 81, 73-97. Retrieved from : https://doc.rero.ch/record/17447/files/Moser-Mercier_Barbara_-_Remote_interpreting_The_crucial_role_20100310.pdf.

Mouzourakis, P. (1996). Videoconferencing: Techniques and challenges. Interpreting, 1 (1), 21-38, https:// doi.org/10.1075/intp.1.1.03mou.

Mouzourakis, P. (2006). Remote interpreting: A technical perspective on recent experiments. Interpreting, 8 (1), 45-66, https://doi.org/10.1075/intp.8.1.04mou.

Peterc, U. (2020). Introduction by the AIIC President. TFDI Newsletter: Issue 1. Retrieved from : https:// aiic.org/company/roster/companyRosterDetails.html?language $=$ fr_FR\&companyId $=11789 \&$ companyRosterId $=26$.

Pöchhacker, F. (1994). Simultandolmetschen als komplexes Handeln (Language in Performance 10). Tübingen : Narr. 
Poyatos, F. (1987). Nonverbal Communication in Simultaneous and Consecutive Interpretation: A Theoretical Model and New Perspectives. TEXTconTEXT, 2-2/3, 73-108.

Poyatos, F. (1997). The reality of multichannel verbal-nonverbal communication in simultaneous and consecutive interpretation. In F. Poyatos (ed.), Nonverbal communication and translation: New perspectives and challenges in literature, interpretation and the media (pp. 249-282). Amsterdam/Philadelphia : John Benjamins, https://doi.org/10.1075/btl.17.

Rennert, S. (2008). Visual Input in Simultaneous Interpreting. Meta, 53 (1), 204-217, https://doi. org/10.7202/017983ar.

Roziner, I. \& Shlesinger, M. (2010). Much ado about something remote: Stress and performance in remote interpreting. Interpreting, 12 (2), 214-247. DOI : 10.1075/intp.12.2.05roz.

Sainsbury, Ch. (2020). The impact of lexical density in simultaneous interpreting of slide presentations: A replication. Master : Univ. Genève.

Seeber, K.G. (2017). Multimodal Processing in Simultaneous Interpreting. In J.W. Schwieter, A. Ferreira (eds.), The Handbook of Translation and Cognition. DOI : 10.1002/9781119241485.ch25.

Seeber, K.G., Kerzel, D. (2011). Cognitive load in simultaneous interpreting: Model meets data. International Journal of Bilingualism, 16 (2), 228-242. DOI : 10.1177/1367006911402982.

Seeber, K.G., Keller, L., Amos, R. \& Hengl, S. (2019). Expectations vs. experience: Attitudes towards video remote conference interpreting. Interpreting, 21 (2), 270-304. DOI : 10.1075/intp.00030.see.

Tomaszkiewicz, T. (2006). Przekład audiowizualny. Warszawa : Wydawnictwo Naukowe PWN. 